\title{
Measurement of cross-counter leader fractions in an 18NM64: Detecting single and multiple atmospheric secondaries
}

\author{
Alejandro Sáiz*, Warit Mitthumsiri, David Ruffolo \\ Department of Physics, Faculty of Science, Mahidol University, Bangkok 10400, Thailand \\ E-mail: alejandro.sai@mahidol.ac.th, warit.mit@mahidol.ac.th, \\ david.rufemahidol.ac.th
}

Paul Evenson

Bartol Research Institute, University of Delaware, Newark, DE 19716, USA

E-mail: evensoneudel.edu

\section{Tanin Nutaro}

Department of Physics, Faculty of Science, Ubon Ratchathani University,

Ubon Ratchathani 34190, Thailand

E-mail: tnutaro@yahoo.com

\begin{abstract}
The Princess Sirindhorn Neutron Monitor (PSNM) is an 18NM64 (with 18 counter tubes in a continuous row) at $2560 \mathrm{~m}$ altitude in Doi Inthanon, Thailand. In late 2015, the electronics in the PSNM were upgraded so as to return an absolute time, referenced to the GPS time, for each neutron count recorded. The timing accuracy is approximately $\pm 3 \mu \mathrm{s}$. This permits extending the well-known concept of multiplicity (multiple correlated counts in one detector) to what we refer to as 'cross multiplicity' or correlation of counts in one detector with those in another. At present, the data are recorded as cross-counter time-delay histograms with 5- $\mu$ s bins. A crosscounter relative leader fraction $L_{i j}$ can be defined as the fraction of pulses measured in counter number $i$ that are uncorrelated in time with the next pulse in counter $j$. For simplicity, we derive average cross-counter leader fractions $L_{\Delta}$ with $\Delta=|i-j|$ as a function of counter tube separation, from a tube separation $\Delta=0$ (i.e., using the same tube) to $\Delta=17$. For low tube separation, $L_{\Delta}$ rapidly increases with increasing $\Delta$, as expected from the spatial spreading of neutrons produced by the same atmospheric secondary particle inside the neutron monitor. At high tube separation, $L_{\Delta}$ increases slowly with increasing $\Delta$ and remains different from unity, which we interpret as due to multiple atmospheric secondaries arriving in rapid succession after originating from the same primary cosmic ray.
\end{abstract}

35th International Cosmic Ray Conference

10-20 July, 2017

Bexco, Busan, Korea

${ }^{*}$ Speaker. 


\section{Introduction}

Neutron monitors (NMs) are ground-based detectors of 'secondary' cosmic rays (mostly neutrons) that are produced in Earth's atmosphere by interactions of ('primary') cosmic rays with energies $\sim 1 \mathrm{GeV}$ and above. In an NM, a number of gas-filled proportional counters operate inside several tons of $\mathrm{Pb}$ (the producer) to detect individual 'tertiary' neutrons produced from the collisions of atmospheric secondary cosmic rays with $\mathrm{Pb}$ nuclei. The rate of detection, and its variations, can then be related to the flux of primary cosmic rays arriving to Earth.

The Princess Sirindhorn Neutron Monitor (PSNM) is located on the top of Doi Inthanon, Thailand's highest mountain, at longitude $18.59^{\circ} \mathrm{N}$, longitude $98.49^{\circ} \mathrm{E}$, and $2560 \mathrm{~m}$ above sea level. The geomagnetic cosmic ray cutoff there is higher than at any other NM location around the world. The main detector contains 18 neutron counters in a continuous row following the NM64 design [1]; 3 other counters operate outside the $\mathrm{Pb}$ producer (bare counters).

Aiming to have a measurement of cosmic ray energies as well as flux, the data acquisition system at the PSNM records also the time of each neutron detection. The distributions of time delays, or times between consecutive neutron detections, can be analyzed to extract a leader fraction $L$ that quantifies the number of detections that are statistically uncorrelated with the previous detection, relative to the total [2]. Typically, two neutron detections that originate from two different cosmic rays are uncorrelated in time as in a Poisson process; on the other hand, tertiary neutrons produced by the same atmospheric secondary in the $\mathrm{Pb}$ are detected within a few $\mathrm{ms}$ and statistically correlated. Thus $L$ can be shown to be related to the energy spectrum of cosmic rays, since production in the $\mathrm{Pb}$ increases for higher cosmic ray energy.

In our previous work [2,3], time delay distributions were studied for consecutive detections in the same neutron counter, and $L$ was calculated for each of the 18 counters independently. Here we note that neutrons produced together in the $\mathrm{Pb}$ (from the same secondary) often get detected by different counters. Also, some time correlation may arise from the detection of different atmospheric secondaries that were produced together in the atmosphere (i.e., from the same primary). For the sake of understanding these processes, in late 2015 the electronics in the PSNM acquisition system were upgraded to allow for cross-counter time delay analysis. In this work, we introduce the technique that allows us to study time correlations between neutrons detected in different counters, we define the appropriate cross-counter leader fractions, and we present some preliminary results of their measurement at the PSNM.

\section{Absolute times in an NM}

To allow for time calculations, the electronics on each neutron counter tube runs an oscillator at $3686.40 \mathrm{kHz}$ to define a sequence timer. When a new detection pulse is registered, its time is recorded based on the sequence timer. A time delay between consecutive pulses recorded in the same counter tube can be easily computed from the difference between values of the sequencer; this technique has been used in the PSNM since it started operating in 2007 [2]. However, since the oscillators on different counters are not synchronized, and the main data acquisition system needs to 'read' the data from each counter sequentially, the values of the sequencer for two different counters are not enough to compute a cross-counter time delay. 
To circumvent this difficulty, the new system deployed in late 2015 uses a once-per-second signal, based on GPS time, sent simultaneously from the main system to all the counters; each counter then records the value of its sequencer corresponding to that synchronization signal. When the data acquisition software receives the data, it can then convert the value of the sequencer of each counter into a GPS time; for stability, this is done once per minute by a fit to the once-persecond values received during the last minute. The achieved timing accuracy is estimated to be approximately $\pm 3 \mu$ s.

\section{Distributions of cross-counter time delays}

Once every neutron detection is assigned a GPS time, any desired time delay can be computed simply as a difference. In this work, we define a cross-counter time delay $t$ from counter $i$ to counter $j$, for each detection in counter $i$, as the time until the earliest detection in counter $j$. Let $R_{i j}(t)$ be the 'survival' probability that no event on counter $j$ has arrived within time delay $t$ after the event of interest on counter $i$. In analogy with [2], we have

$$
R_{i j}(t)=\exp \left(-\alpha_{j} t\right) \exp \left[-\int_{0}^{t} \beta_{i j}\left(t^{\prime}\right) \mathrm{d} t^{\prime}\right],
$$

where $\alpha_{j}$ is the probability per time of a chance coincidence in counter $j$, and $\beta_{i j}\left(t^{\prime}\right)$ is the probability of a temporally related count on $j$ after time delay $t^{\prime}$. (While there is no direct electronic dead time to prevent registration of a count on a different tube $j$ after a count on $i, \beta_{i j}\left(t^{\prime}\right)$ does include indirect effects of the dead time. For example, if a count on $i$ immediately followed a count on $j$, then $j$ would be unable to register a new count for $\sim 80 \mu$ s.) At a time $t$, after a few ms, $\beta_{i j}\left(t^{\prime}\right)$ is very close to zero (i.e., the neutron counts associated with the same primary CR have all been collected) and the second exponential tends to a constant value that we call the leader fraction $L_{i j}$, i.e., the probability that a count on $i$ is not temporally associated with any later count on $j$. Then the normalized distribution of time delays $n_{i j}$ is given by

$$
n_{i j}(t)=-\frac{\mathrm{d} R_{i j}}{\mathrm{~d} t}
$$

At long times, the exponential tail of the normalized distribution can then be expressed as

$$
n_{i j}(t) \rightarrow \alpha_{j} L_{i j} \exp \left(-\alpha_{j} t\right) \quad t>t_{\text {trans }}
$$

for a transition time $t_{\text {trans }}$ of a few ms.

To reduce the number of recorded distributions and increase the statistics, in this work we analyze the combined distributions of cross-counter time delays at a given counter separation $\Delta$, or $n_{\Delta}(t)$, with $\Delta=|i-j|$, for $\Delta=0, \ldots, 17$. The case $\Delta=0$ of zero separation is the same-tube time delay distribution studied in our previous work, combined for the 18 counters. Some examples of (unnormalized) distributions are shown in Figure 1. These distributions are recorded both as shorttime histograms (with bin size of about $4.9 \mu$ s for $t<5 \mathrm{~ms}$ ) and long-time histograms (with bin size of about $0.98 \mathrm{~ms}$ up to $t=1 \mathrm{~s}$ ). In Figure 1 the transition from short-time to long-time histogram is visible at $t=5 \mathrm{~ms}$. The dashed lines indicate each of the exponential functions that best fit the longtime histogram tail up to $200 \mathrm{~ms}$. Although most prominent at the smaller values of $\Delta$, the peak 
at small $t$ is evident at every separation, indicating that there are some counts that are temporally associated even at the maximum separation of 17 , from counter 1 to counter 18. Our interpretation is that the large cross-counter multiplicity seen at small separation is due to interactions inside the NM that produce multiple neutrons and counts on nearby counters. However, for counters at large separation, there is a nearly constant residual cross-counter multiplicity that we attribute to multiple atmospheric secondaries from the same cosmic ray that lead to counts on different random counters.

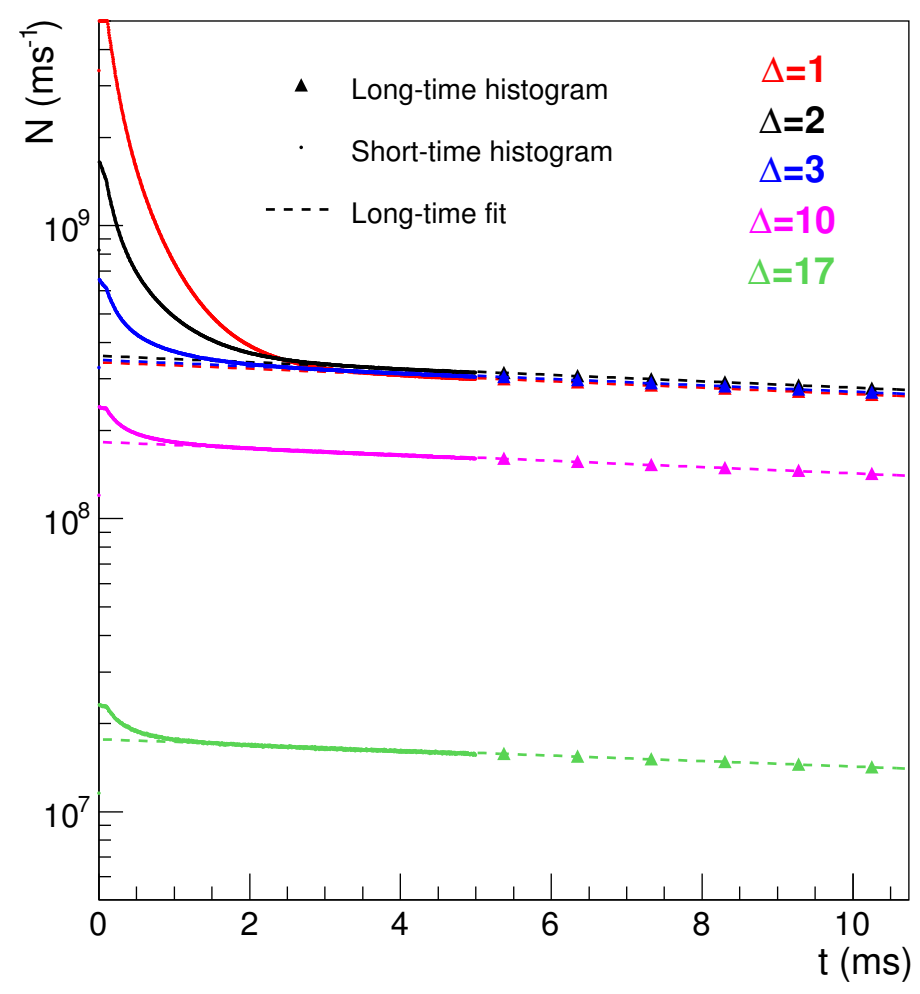

Figure 1: Unnormalized distributions of cross-counter time delays $t$ at counter separation $\Delta$ of 1, 2, 3, 10, and 17. Each distribution comes from histograms of $t$ accumulated for $\sim 4800 \mathrm{~h}$. Dashed lines are exponential fit functions to the data between 10 and $200 \mathrm{~ms}$.

\section{Calculation of cross-counter leader fraction}

We define the cross-counter leader fraction at separation $\Delta, L_{\Delta}$, by combining all combinations $i j$ of counters with $|i-j|=\Delta$. Assuming the values of $\alpha_{j}$ are similar for all $j$, the equation for the exponential tail then becomes

$$
n_{\Delta}(t) \rightarrow \alpha L_{\Delta} \exp (-\alpha t) \quad t>t_{\text {trans }}
$$

We can use this expression to estimate $L_{\Delta}$ from hourly histograms of $t$ by fitting an exponential function to some interval where $t>t_{\text {trans }}$.

To study the overall properties of $L_{\Delta}$ with good accuracy, in this work we add together several months of hourly data before estimating $L_{\Delta}$. To improve the fit, we remove the hours when the value 
of $\alpha$ deviated from the mean ( $\alpha$ varies due to changes in atmospheric pressure), so the resulting accumulated histograms contain $\sim 4800 \mathrm{~h}$ of data. For each of the 18 histograms $(\Delta=0, \ldots, 17)$, an exponential function is fit to the $t$ interval $10-200 \mathrm{~ms}$. The two parameters from the fit give $\alpha$ and $L_{\Delta}$ after proper normalization.

\section{Results}

Figure 2 shows the cross-counter leader fraction $L_{\Delta}$ as a function of counter separation $\Delta$. The values start at less than 0.8 for separation zero (same counter) and quickly increase when considering cross-counter time delays between distant counters, approaching a constant value close to 1 . A value of $L_{\Delta}=1$ would correspond to totally uncorrelated neutron detections between counters (a Poisson process). However, the value of $L_{\Delta}$ that is observed, which seems to vary little for separations $6-17$, is never consistent with 1 . We can also see that it cannot be 1 from Figure 1, where the peak at short $t$ is visible above the exponential tail for all separations. A fit to the combined histogram including all separations larger than 5 gives a value $L_{\Delta>5}=0.9973$.

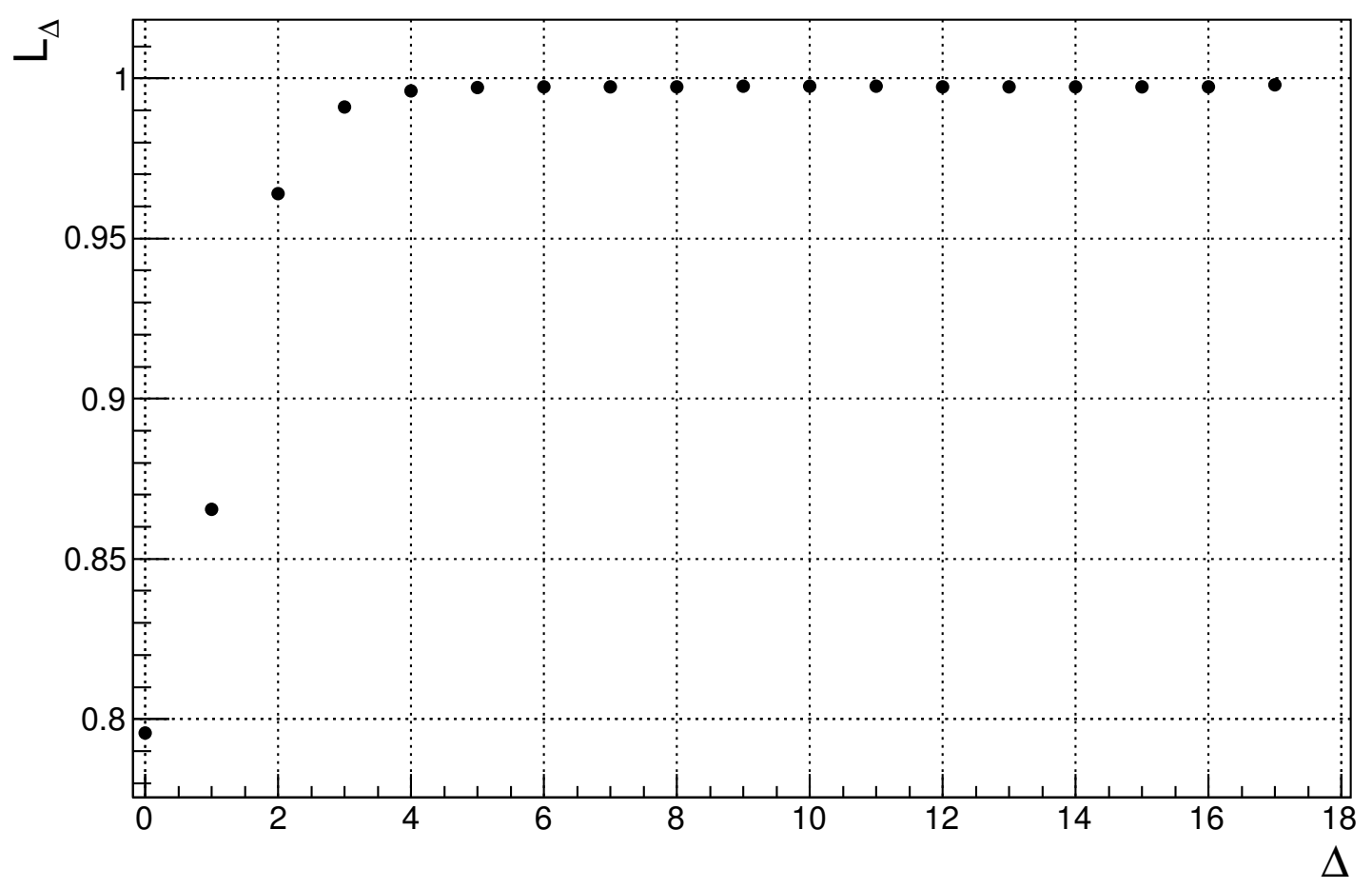

Figure 2: Cross-counter leader fraction $L_{\Delta}$ as a function of counter separation $\Delta$.

For small separation $\Delta$, we can understand the leader fraction (inverse of the multiplicity) as coming from the production of multiple tertiary neutrons in the $\mathrm{Pb}$ producer, which subsequently travel inside the NM before being detected by two different counters. The variation of $L_{\Delta}$ with $\Delta$ is also consistent with that idea, since there is a smaller probability for a neutron to travel a longer distance inside the NM between the point of production and the point of detection. However, for large separations, propagation of tertiary neutrons inside the NM is not a satisfactory explanation because of the constancy of the value of $L_{\Delta}$. The most plausible mechanism that could produce 
values of $L_{\Delta}$ different from 1 and independent of $\Delta$ is the arrival of two different atmospheric secondary particles that are correlated in time. Secondary cosmic rays are always part of a particle cascade that originates from a single primary cosmic ray and that can extend tens or hundreds of $\mathrm{km}$ when it reaches the ground. Thus we would expect the spatial correlation between secondaries to depend on distance negligibly at these scales (counter to counter separation is $0.5 \mathrm{~m}$ ) and two of these secondaries could arrive to the NM with probability that is independent of $\Delta$.

We therefore interpret the value $\sim 0.997$ of the cross-counter leader fraction at large counter separation as an atmospheric secondary leader fraction between two counters, which quantifies a correlation between particles of the same atmospheric cascade and that should be present at any distance, and even when no tertiary neutrons are involved. One example, but difficult to test, would be two NMs separated by several $\mathrm{km}$. Another case that could possibly present this atmospheric leader fraction less than 1 is a bare counter. At PSNM, there are three 'bare' counters that are not surrounded by $\mathrm{Pb}$ producer (or polyethylene reflector), and the neutrons they detect may include both secondary (atmospheric) neutrons and tertiary neutrons produced from materials in the environment. To test this idea we also calculated the (same-counter) leader fraction $L$ for the 3 bare counters in PSNM, and we find values between 0.996 and 0.998 . This suggests that multiple atmospheric secondaries may play a major role in the multiplicity of bare counters.

Considering the entire NM, with 18 counters, the secondary leader fraction can be estimated as $L_{\Delta>5}^{18}=0.953$. In other words, considering the entire NM, $95.3 \%$ of the counts did not follow another count from the same primary cosmic ray and a different atmospheric secondary particle, while $4.7 \%$ of the counts did. If we make the additional assumption that energy of the atmospheric secondary is independent of the probability that another secondary produced a count somewhere in the NM, i.e., that probability is independent of the multiplicity due to a given secondary, then we can estimate that $4.7 \%$ of the atmospheric secondaries that produced counts in the NM were accompanied by another secondary that also produced counts in the NM. The secondary leader fraction, estimated as 0.953 , represents the inverse of the secondary multiplicity, estimated as 1.049.

In previous work, we have shown that the same-counter leader fraction serves as a measure of the Galactic cosmic ray spectral index, and used it to identify short-term spectral variations during Forbush decreases due to solar storms as well as long-term spectral variations due to modulation with the solar activity cycle $[2,3,4]$. The atmospheric secondary leader fraction should be used to correct this same-counter leader fraction when comparing with Monte Carlo results for individual atmospheric secondaries. In further work, we will study whether the cross-counter leader fraction can also serve as an estimate of the cosmic ray spectral index, and whether it provides distinct information from the same-counter leader fraction in order to further constrain the cosmic ray spectrum.

\section{Acknowledgments}

Partially supported by the Thailand Research Fund award RTA5980003, TRG5880173, and US National Science Foundation awards PLR-1245939 and PLR-1341562 and their predecessors. 


\section{References}

[1] C. J. Hatton and H. Carmichael, Experimental investigation of the NM-64 neutron monitor, CaJPh 42, 2443 (1964).

[2] D. Ruffolo, A. Sáiz, P.-S. Mangeard, N. Kamyan, P. Muangha, T. Nutaro, S. Sumran, C. Chaiwattana, N. Gasiprong, C. Channok, C. Wuttiya, M. Rujiwarodom, P. Tooprakai, B. Asavapibhop, J. W. Bieber, J. Clem, P. Evenson and K. Munakata, Monitoring short-term cosmic-ray spectral variations using neutron monitor time-delay measurements, ApJ 817, 38 (2016).

[3] P.-S. Mangeard, D. Ruffolo, A. Sáiz, W. Nuntiyakul, J. W. Bieber, J. Clem, P. Evenson, R. Pyle, M. L. Duldig and J. E. Humble, Dependence of the neutron monitor count rate and time delay distribution on the rigidity spectrum of primary cosmic rays, JGRA 121, 11620 (2016).

[4] C. Banglieng, D. Ruffolo, A. Sáiz, P. Evenson and T. Nutaro, Tracking cosmic-ray spectral variations with neutron monitor time-delay measurements at high cutoff rigidity during 2007-2017, these proceedings. 